\title{
Espace de travail et logique documentaire
}

Workspace and Documentary Logic

Dominique Cotte

\section{(2) OpenEdition \\ Journals}

Édition électronique

URL : http://journals.openedition.org/edc/436

DOI : $10.4000 /$ edc.436

ISSN : 2101-0366

Éditeur

Université Lille-3

\section{Édition imprimée}

Date de publication : 1 octobre 2007

Pagination : 25-38

ISBN : 978-2-9514961-9-4

ISSN : 1270-6841

Référence électronique

Dominique Cotte, "Espace de travail et logique documentaire », Études de communication [En ligne], 30 | 2007, mis en ligne le 01 octobre 2009, consulté le 19 avril 2019. URL : http://

journals.openedition.org/edc/436 ; DOI : 10.4000/edc.436

Ce document a été généré automatiquement le 19 avril 2019

(C) Tous droits réservés 


\title{
Espace de travail et logique documentaire
}

Workspace and Documentary Logic

\author{
Dominique Cotte
}

\section{Introduction}

1 Vers 1910, aux États-Unis, une véritable révolution se produit dans le travail de bureau : l'invention du dossier suspendu pour classer les documents. Jusqu'alors, coexistaient deux façons d'organiser la documentation de travail : l'enregistrement dans des livres pour le repérage des pièces et le classement par piles dans des meubles à casiers. Le dossier suspendu, mode de classement spatial, permet de réunir dans un même ensemble les différentes pièces d'un dossier en les hiérarchisant, par exemple un dossier client et autant de sous-dossiers que d'éléments de l'affaire (commandes, factures, courrier, contentieux...), chose que ne permettait pas l'enregistrement chronologique de l'arrivée des pièces, par définition discontinu. Ce passage a été étudié par JoAnne Yates (Yates, 1982), une chercheuse américaine du M.I.T., spécialisée dans le management ${ }^{1}$.

2 Je la cite en ouverture de cet article pour deux raisons :

- tout d'abord pour signifier que notre discipline, les Sciences de l'information et de la communication [Sic], sont capables de s'intéresser à l'influence d'objets relevant de la plus ordinaire quotidienneté, pour en examiner les impacts à la fois organisationnels, sociologiques, et bien sûr communicationnels et sémiotiques; c'est la même logique qui nous fait nous intéresser, dans le interfaces des écrits d'écran et des architextes ${ }^{2}$, au moindre signe énoncé, comme par exemple la flèche, ou l'icône du dossier suspendu (justement) qui figure comme métaphore du classement de nos fichiers sur nos disques durs ;

- ensuite afin de montrer, à travers cet exemple si ordinaire et tellement parlant (nous avons tous, dans une armoire ou un tiroir de nos bureaux cet objet qui paraît si naturel et qui pourtant, donc, possède aussi une histoire), que document et espace sont deux notions qui 
naturellement, ont partie liée. Et ceci doublement : un document est en soi un espace sur lequel sont disposés des signes pour organiser la communication; et ensuite, un document se range - en principe au moins ! - et pour cela il lui faut trouver un espace idoine, lequel vaut à la fois comme arrangement physique et comme organisation intellectuelle.

Or, nous avons affaire depuis une dizaine d'années, à ce que d'aucuns nomment la révolution numérique, qui n'est en fait que l'aboutissement de la promesse de l'informatique. Ce phénomène est faussement interprété comme un mouvement de dématérialisation ${ }^{3}$, à la fois du document et des espaces de travail et de communication. Or, comme le défend au contraire une partie des chercheurs de notre discipline, la logique documentaire, fut-elle numérique, reste profondément ancrée dans un univers de matérialité, fait de machines, de flux électriques, de réseaux et de câbles, sans compter bien sûr les imprimantes qui restent encore de fidèles alliées de la lecture. Mais ce qui est vrai c'est que ce changement de régime de matérialité appelle des recompositions nombreuses, lesquelles passent par des changements d'angle, de nouvelles représentations, et le recours à des métaphores puissantes pour symboliser différemment un espace dans lequel le corps ne se meut plus directement, mais qui occupe les écrans de travail de nos ordinateurs et dans lequel il faut, malgré tout, se repérer. En place de matérialisation, il conviendrait de parler de tangibilité car il est vrai que le fait de ne plus pouvoir toucher directement le document en tant qu'objet et le voir matérialisé sous forme de signe, d'icône, de représentation, change fondamentalement la donne. C'est à partir de cette idée de représentation qui vient remplacer le document et l'espace tangible que nous proposons ici de voir comment la logique documentaire structure ces nouveaux espaces de travail informatisés.

\section{L'espace du document et ses avatars}

4 Tout document peut être conçu comme un espace visant à organiser l'information. En tant que support sémiotique, c'est-à-dire surface organisée pour produire du sens, le document se distribue selon une logique spatiale, sur toute la surface de ce support, selon des canons qui varient au cours de l'histoire, en fonction d'une part des possibilités techniques existantes pour une époque donnée (par exemple on roule le papyrus parce que la surface de la feuille trop cassante, ne peut pas être pliée), et d'autre part des modes culturels d'appréhension des formes et contenus documentaires, les deux aspects étant bien sûr étroitement liés. En même temps, Annette Béguin montre (Béguin, 2006) que ces modes culturels s'oublient si bien qu'ils finissent par (nous) paraître naturels; ainsi des bordures et des cadres qui sont là pour structurer l'espace de l'information, mais qui ont l'art de se rendre invisibles. De son côté, Yves Jeanneret rappelle pour sa part que les textes sont soumis à un « impératif de lisibilité » qui les ramène toujours à une logique de la forme et qui amène les formes à se coter entre elles pour que le lecteur puisse trouver des points de repères (Jeanneret, 2001).

Cela a été tout l'art des imprimeurs humanistes, après celui des scribes et des moines copistes que d'arranger, en fonction des moyens existants qu'ils étaient capables de manipuler, et dans le cadre des représentations qui étaient les leurs, d'organiser des surfaces documentaires pour assurer une certaine forme de présentation des savoirs dans une société donnée. Les typographes de l'époque moderne et contemporaine n'ont ensuite pas cessé de continuer à expérimenter, innover, chercher, tout en s'essayant à reproduire des formes efficaces parce que reconnues (André, paccaud, 2004). 
6 En ce sens, nous avons là un des premiers rapports logiques et conceptuels, en même temps que pratique, du document à l'espace. C'est l'espace de la feuille, qui devient page (Souchier, 1993), l'espace de la marge, qui permet la respiration du texte, l'espace organisé des pages de titre des ouvrages, des «Unes» des journaux, qui permet de hiérarchiser l'information.

7 Mais l'espace du document n'est pas que plan, il est aussi en trois dimensions, volumique. Les imprimeurs ont su utiliser les dos, ainsi que les couvertures des volumes pour en faire des espaces d'information en même temps que des éléments décoratifs, mais qui s'avèrent également, en tant que tels, signifiants (par exemple une collection chez un éditeur, joue sur les bandes présentes sur les dos des volumes pour présenter un élément visuel reconnaissable dans une bibliothèque). La gestion du volume est rarement étudiée par les chercheurs, alors qu'elle nous semble un élément essentiel de la dimension documentaire, ne serait-ce que parce qu'elle organise l'acte de lecture par le truchement d'un des organes qui, anthropologiquement caractérise fondamentalement l'espèce humaine: la main (le cerveau ne venant, à mon sens, qu'en second). Dans l'acte de feuilletage d'un livre, d'un magazine, d'un dossier documentaire, d'une pile de papier, se réalise une subtile prise de connaissance par le biais d'une sélection rapide des éléments qui vont aider le lecteur à effectuer cette semissis qui résulte, normalement, de la consultation d'un document.

8 Distribué dans l'espace du volume, le document se dote d'éléments de repérages en quelque sorte endogènes, comme par exemple la mise en chapitres, la pagination, l'organisation en cahier pour les magazines et les journaux, la présence de sommaires, d'index, de tables des matières. Tous ces éléments de structuration intellectuelle sont aussi des outils de repérage spatiaux, tout comme la carte permet de se repérer sur le territoire.

9 Enfin, on pourrait dire sans provocation excessive que le document, au sens d'élément isolé, unique, n'existe pas. Tout document se trouve relié à d'autres, soit par un effet de collection (tous les journaux quotidiens d'un titre sur une année par exemples), soit par des effets d'intertextualité (par exemple les écrits de commentaires d'un texte classique), soit par des logiques administratives (la déclaration d'impôts remplie par le contribuable et les documents déclaratifs de l'employeur qui voisinent dans le dossier de l'administration fiscale). De ce point de vue, les documents se retrouvent liés les uns aux autres dans des espaces qui sont cette fois des contenants : boites, dossiers, étagères, armoires, pièces de bureau, salles de lecture, réserves, édifices des bibliothèques, en allant du plus petit au plus vaste de ces espaces. Cependant, ces contenants, éléments de repérage cette fois exogènes, sont eux-mêmes des ensembles sémiotiques car ils « disent » quelque chose sur les documents contenus, grâce à leur positionnement, leur classement, leur répartition dans l'espace général. Les bibliothèques en accès libre, qui sont aujourd'hui la norme, organisent la circulation des lecteurs à travers une représentation thématique qui épouse les contours de l'organisation physique des travées et des étagères. L'utilisation des grands systèmes de classification dits " universels » comme la CDU ou la Dewey pour organiser cette circulation concourt à transformer l'espace physique en un espace signifiant.

10 Or, l'évolution contemporaine $\mathrm{du}$ document, vers des formes numériques ou électroniques contribue à déstructurer ce rapport à l'espace documentaire : dans le plan, dans le volume et au sein d'un contenant plus général. Sans pouvoir ici rentrer dans les détails $^{4}$ car le sujet est très vaste, on constatera que ce qui définissait d'une certaine 
manière l'unité documentaire (la fixité, l'identification par l'objet, l'autorité...) est considérablement modifié dans le cadre de la numérisation des process qui aboutissent tant à la fabrication du document qu'à sa mise en circulation et sa mise en lecture.

11 Dans le cadre de l'évolution du document en général vers le numérique, la question de la relation à l'espace se pose doublement, car le document - lu en général sur un écran - ne peut pas être détaché des artefacts et dispositifs qui en permettent d'un côté la lecture, de l'autre le classement. La caractéristique d'un livre emprunté momentanément à une bibliothèque est qu'il entre et sort du dispositif selon les moments et les usages; dans une poche, dans une sacoche, sur une table de travail, il ne fait momentanément plus partie de l'espace organisé qui est le sien dans le cadre de la bibliothèque. A l'inverse, le document numérique - tant qu'on ne l'a pas imprimé une nouvelle fois - est inséparable de son architexte, c'est-à-dire du dispositif qui permet de l'organiser, de l'afficher, de le lire, d'agir dessus - par exemple en cliquant sur une partie, sur un lien -. Ce que l'on présente au lecteur à l'écran, c'est une double simulation de l'espace : l'espace sémiotique du document lui-même, et l'espace d'organisation du dispositif à l'intérieur duquel se trouve classé, rangé, décrit, indexé, le document en question.

Une fois posé ce cadre général, on peut s'intéresser à une variante spécifique, qui sort du monde classique de la documentation organisée dans des structures telles que les bibliothèques, les services de documentation, les centres d'archives, pour examiner comment évolue le rapport du document à l'espace dans le contexte du travail quotidien : bureaux, usines, entreprises, administrations sont des lieux où des salariés sont payés pour effectuer des tâches, mais ce sont également des lieux d'écriture et des espaces où circulent en quantités considérables des documents de toutes sortes : notes, rapports, comptes-rendus, manuels, factures, écrits techniques, plans, listes, procédures, consignes, livres, journaux, courriers... sous une forme aussi bien imprimée que numérique. L'informatisation croissante des processus de travail accentue ce phénomène de textualisation (Despres, Cotte, 2005), car ce que l'individu effectuait auparavant par le geste, il doit le simuler à l'écran au moyen de la machine. Ainsi, les intranets, ou les portails d'entreprise par exemple, représentent autant d'architextes qui servent quotidiennement à la manipulation de données, d'informations, mais aussi de documents. Le rapport traditionnel à l'espace de travail comme lieu d'organisation et de classement s'en trouve profondément modifié.

\section{L'espace de travail comme lieu de classement}

13 Au milieu des années 1990, deux chercheurs (Fischler, Lalhou, 1995) analysent pour EDF les comportements des salariés en matière de gestion de leurs documents de travail, dans le contexte "situé » de leur environnement immédiat. L'association de Claude Fischler, anthropologue spécialisé dans les questions liées au goût et à l'alimentation, et de Saadi Lahlou, chercheur en sciences cognitives à EDF produit la métaphore organique stimulante de la digestion de l'information. Dans cette perspective «le bureau » est vu comme un organisme vers lequel confluent des éléments disparates : documents, objets de travail, informations, qu'il convient, non pas de «traiter» comme le dirait un professionnel de l'information, mais de " gérer et digérer », comme le fait un organisme dans une action qui n'est pas strictement contrôlée, qui suit des règles naturelles. De ce fait, c'est un ensemble d'organes qu'il convient d'étudier, car l'individu n'est pas autonome face à « l'information », il est situé, dans un ensemble disparate ainsi décrit : 
Ainsi, il apparaît que le consommateur d'information ne peut être réduit à un individu: il s'agit en fait d'un individu intégré dans un ensemble fonctionnel, comprenant son bureau (meuble et pièce), son équipement de rangement et de stockage, ses outils de communication, ses collaborateurs proches, ses supérieurs et subordonnés immédiats, etc. En termes éthologiques et spatiaux, cet ensemble fonctionnel relève de la notion de territoire : il est sous le contrôle partie du sujet, en interaction avec les autres individus de son milieu professionnel. En termes écologiques, il est constitué d'un ensemble d'interactions avec les sous-ensembles voisins, qu'il s'agit d'appréhender sans le mutiler, en y incluant autant que possible les boucles de rétroaction multiples entre les différents éléments, sous-systèmes et systèmes concernés (individu, bureau, collègues, organisations, etc.) [...]. un ensemble composé du travailleur et de son espace de travail, éventuellement de ceux avec qui il le partage, des lieux réels ou virtuels de stockage ou de partage de l'information pertinente pour la tâche ou des outils nécessaires à son exécution (local de photocopie ou d'archivage, éventuellement une partie du cybermonde. Nous appelons cet ensemble la « cellule de travail».

Pour nos deux auteurs, l'environnement de travail est un « milieu » exactement comme en écologie une espèce s'observe au sein de son écosystème. Face à un flux constant et croissant de documents de toute nature, il s'opère une forme de gestion spontanée qui consiste en la fabrication de piles, de dossiers... Dans leur étude, Fischler et Lalhou montrent l'importance de la distribution spatiale des documents dans l'espace du bureau, ce qu'ils appellent «l'ergotope »:

- il existe un contact sensoriel avec l'information à chaque instant (ceci est du à la tangibilité du document dans le contexte analogique, et cette tangibilité fait intervenir des éléments tels que la couleur, l'épaisseur, le vieillissement... qui disparaissent dans l'univers de la représentation numérique et sont représentés de manière métaphorique ;

- la proximité spatiale, par exemple la localisation dans la hauteur de la pile ou par rapport à la situation de l'individu (plus ou moins loin de sa situation dans l'espace de travail) possède une signification quant au niveau d'urgence ou d'importance du document ;

- l'importance de la possibilité de manipulation physique : «Ce qui est important doit être à portée de main et/ou à portée de regard [...], l'opposition proximal/distal correspond à une dimension temporelle, à l'opposition urgent/peut attendre ».

15 Ceci montre, pour le traduire dans un langage sémiotique, qu'un espace de travail, ergonomiquement organisé pour faciliter l'organisation de la tâche, est aussi un espace saturé de signes qui permettent à l'individu de se repérer dans la masse documentaire qui le submerge. Deux phénomènes ainsi se croisent: d'une part, la complexification croissante des univers de travail, la textualisation des pratiques, l'amplification de la masse documentaire, qui pose à l'individu au travail des problèmes d'organisation et de repérage cognitif; d'autre part, l'invention, pour pouvoir résoudre ces problèmes, de dispositifs spontanés de la part des individus, qui lui permettent de se repérer dans cette masse de documents qui sont aussi des outils de travail. Il se pratique ainsi une forme d'organisation spontanée de l'espace documentaire qui ne ressemble certes pas à celle qui est recommandée par les spécialistes de la discipline, les documentalistes, les archivistes, mais qui n'en est pas pour autant inefficace.

Cette saturation de l'espace de travail par des signes a également été relevée par une partie des membres de l'équipe de recherche mobilisée autour d'une étude pour la Bibliothèque publique d'information, sur les nouvelles pratiques de lecture/écriture. Joëlle le Marec et Igor Babou (Le Marec, Babou, 2003) observent la façon dont, autour de la focalisation sur les écrans de travail organisés sur la base d'une application 
informatique orientée vers le cœur de métier (en l'occurrence le catalogage) s'organise tout un système de communication mêlant différents éléments : consignes données par courriel imprimées puis affichées sur des panneaux, volumes imprimés de la norme présents sur les bureaux, post-it... Ils appellent ces ensembles des composites.

Il serait faux de considérer tous ces différents éléments (la pile chez Fischler et Lalhou, le post-it chez Le Marec et Babou), comme des sortes de perversions du système ou des manières de détournements. L'idée d'un braconnage ou d'une adaptation tactique par rapport à des stratégies établies par d'autres, telle qu'elle est défendue par un auteur comme Michel de Certeau possède une certaine force, mais elle risque aussi d'entériner une césure artificielle entre ce qui relèverait de la norme et ce qui renverrait à un détournement de cette dernière. Si les "usages" se manifestent souvent par le détournement de ce qui était originellement souhaité ou prescrit (Perriault, 1989), on peut aussi penser que l'accomplissement des promesses initiales des systèmes ne peut réellement advenir que par le truchement de ces ajustements qui du même coup, ne sont pas à côté des systèmes, mais en font partie de manière intégrale. De la même manière, la vulgarisation scientifique n'est pas simplement une traduction de savoirs originels qui se transféreraient ensuite vers la sphère publique, mais la science n'existe que par l'entrelacement de ses expressions « pures » et vulgaires (Jeanneret, 1994).

Que se passe-t-il, lorsque ce dispositif, déployé dans l'espace concret et tangible du lieu de travail, se trouve ramassé sous la forme des applications informatiques qui absorbent la plus grande partie de l'activité de l'individu au travail, lorsque ce que l'individu pouvait faire dans son ergotope, au moyen d'une organisation de son espace physique personnel, il est obligé de le reproduire, au moins en partie, dans des dispositifs qui fonctionnent comme des médias?

\section{Les applications informatiques d'entreprise comme métaphores de l'espace social}

19 L'informatique d'entreprise a connu, depuis une trentaine d'années une très forte progression qui lui a permis de s'emparer peu à peu de la quasi-totalité des applications de travail et des organes de communications. L'informatique de gestion des années 1970 ne mettait pas encore le salarié directement en contact avec la machine ou rarement, on est passé aujourd'hui à un ratio d'équipement qui est souvent d'un poste de travail informatisé par salarié, poste de travail vers lequel convergent un grand nombre d'applications, généralistes (comme le courrier électronique par exemple) ou dites " métier " (concernant une tâche particulière ou un ensemble de tâches propres à une des fonctions de l'entreprise). Il existe également une forte interpénétration des technologies entre le « dehors » et le «dedans", « l'extra » et "l'intra » comme le montre le vocable d'intranet forgé pour illustrer l'utilisation des technologies de l'internet au sein de réseau fermé (protocole TCP-IP, langages html et xml, navigateurs utilisés comme interface unique, etc.).

20 L'intégration de ces différentes applications culmine dans la mise en œuvre de ces intranets, des portails d'entreprise ${ }^{5}$, ou encore des ERP (enterprise ressource planners ou PGI, progiciel de gestion intégré en français) qui deviennent l'outil de travail quotidien, pour les salariés, et qui fonctionnent comme de véritables médias. 
21 L'un des axes de recherche sur lesquels nous travaillons est de relier l'analyse de cette évolution, non seulement en termes technologiques, mais également en termes socioorganisationnels et en faisant intervenir une approche sémiotique de la question. En effet, en tant qu'applications informatiques, ou plus précisément en tant qu'architextes, ces applications relèvent de l'analyse des écrits d'écran, sur lesquels se penche une partie de la communauté des Sic depuis quelques années. L'informatique, qui fonctionne par strates (Cotte, 2004), induit en effet une forte composante liée à l'écriture, car elle doit proposer l'accès aux données et aux informations dans des cadres formels qui sont autant d'objets sémiotiques et de dispositifs de lecture; mais il s'agit également - et principalement - d'une sémiotique de l'action, car ce sont des textes qui ne sont pas seulement là pour être lus, mais pour faire et pour faire faire (à la machine). La préemption croissante des tâches par l'informatique aboutit d'une part à une véritable textualisation des pratiques, d'autre part à une explosion documentaire (dans les deux sens du terme : production d'une masse nouvelle et croissante de documents, et besoin de documenter des pratiques et des actions) ${ }^{6}$.

22 Du point de vue de la question qui est discutée ici, celle de la relation entre espace et document, les architextes des portails d'entreprise et autres applications intégrées nous intéressent doublement.

23 D'une part ces outils suppose qu'un individu, même s'il n'est pas rivé en permanence à son écran, travaille essentiellement au sein de cet espace, qui est un espace simulé et métaphorisé ; d'autre part, l'explosion documentaire concomitante de la montée de ces outils et des techniques de numérisation transpose au sein du portail les problématiques déjà affrontées par les professionnels de l'information dans les lieux «classiques » de la documentation en termes de représentation des contenus, de classification des objets, de recherche d'information... Dans les deux cas, la question du repérage dans l'espace se pose, mais il s'agit soit de l'espace physique et social de l'entreprise, avec ses départements, ses services, ses lieux, qui correspondent à des hiérarchies et des rapports de pouvoir subtils, soit de l'espace de gestion documentaire. Les architextes des portails font se rejoindre ces deux espaces en un seul.

24 L'idée du bureau virtuel ou de l'interface commune aboutit à la consultation sur le même plan des brèves d'information, des écrits professionnels, les archives de l'entreprise, une interface de saisie d'informations structurées, les informations institutionnelles, un annuaire...

Les promoteurs de ces outils essaient d'utiliser cette plasticité pour reproduire une forme de proximité que connaissait l'individu lorsqu'il avait à classer lui-même ses documents de travail dans son ergotope. Il est prévu (souvent dans la partie droite de l'écran, ergonomiquement la plus apte à attirer l'œil et le geste du clic) d'offrir à l'intranaute un espace personnalisé, dans lequel il (ou la machine) pourra "classer » les éléments qu'il juge le plus important. L'emploi du pronom personnel est omniprésent: "Mes » documents, « Mes » outils, « Mes groupes ». Nous avons affaire là à un artifice sémiotique qui vise à concilier le point de vue global de l'organisation et le point de vue individuel du collaborateur.

Il est intéressant de constater que cette répartition fictive des espaces à l'écran épouse donc une hiérarchie qui a été pointée dans le troisième texte collectif signé Roger Pédauque (Pedauque, Salaün, 2006) comme constitutive des nouveaux déplacements documentaires : la sphère du je/tu (privé), la sphère du nous (groupe, collectif), et la 
sphère du on (public). On retrouve dans nombre d'applications internes, cette répartition circulaire qui part de l'individu, s'élargit ensuite à un cercle proche qui peut être le département ou le service, pour finalement se placer au niveau de l'organisme. Ainsi, sous la forme d'onglets, ou de rubriques (les portlets dans les applications de portail), ces espaces se définissent par le « Moi » (mon espace de travail, mes mails, mon agenda, mes outils...), et deux niveaux de " nous » qui sont celui de la sphère de travail immédiate (qui pourrait se traduire dans l'espace physique par les collègues partageant le même bureau ou le même couloir), et celui de l'entreprise considérée comme un tout.

Dans la métaphore spatialisante du portail, l'individu a accès à un espace social, mais cet espace est hiérarchisé et, comme dans la vie réelle, il y a des portes qu'on est habilité à franchir, des couloirs qui sont interdits, d'autres auxquels on ne peut accéder qu'après avoir "badgé » ou laissé sa pièce d'identité à une hôtesse d'accueil. La métaphore de la clôture, de la serrure, est omniprésente, souvent représentée par des cadenas, qui autorisent ou non le fait de pénétrer dans un espace ou dans un autre. Ce contrôle de l'espace social, même métaphorisé, renvoie évidemment aux questions du pouvoir dans les organisations (Carmes, 2002).

Par ailleurs, dans la mesure où la logique de la numérisation affecte l'identification des documents et de leurs contenus par les lieux de leur conservation, les portails, qui sont aussi de vastes ensembles documentaires, doivent mettre en place un ensemble d'outils et de structures qui permettent de retrouver les documents. La question de la représentation en amont des contenus passe par la simulation d'un espace documentaire qui est essentiellement langagier: listes de rubriques, nommage d'espaces, titres de documents... Nous retrouvons donc là une autre marque de la logique documentaire car les portails et intranets, en tant qu'écrits d'écran, et en tant que médias, organisent une représentation sémiotique de l'espace de travail en utilisant des procédés et techniques qui sont ceux qui caractérisent l'art documentaire. Les normes relatives, par exemple, au records management, insistent sur les règles de nommage des documents et fichiers, sur la nécessité de plans de classement basés sur la logique de l'activité et sur le partage de ces règles.

\section{Conclusion}

Il ne faudrait cependant pas trop vite souscrire, nonobstant la réalité des phénomènes décrits ci-dessus, à l'idée que, tel un trou noir, l'écran absorbe la totalité de la représentation de l'espace de travail. Si telle est la vision représentée dans les discours auto-légitimants des éditeurs commerciaux et des promoteurs de ces solutions au sein des organisations : webmestres, chefs de projets, responsables informatiques, responsables de communication, on observe malgré tout des stratégies d'évitement, de duplication, de reproduction d'espaces privés en marge de l'espace public qui est rarement totalement contrôlé par la structure. A moins de supposer un retour total vers l'informatique des années 1970, avec un réseau de terminaux distribués autour d'un serveur central, ces dispositifs souffrent de la contradiction entre la logique centralisatrice du réseau, et la logique distribuée de la bureautique des années 1980, renforcée récemment par la logique totalement ouverte du web. Ayant à sa disposition aussi bien son disque dur sur sa machine dans son bureau que des « espaces partagés », « dossiers mutualisés », « dossiers publics » sur les logiciels de partage ou les portails, l'individu choisit bien souvent ce qui lui est le plus commode et le plus inscrit dans ses « routines». Ainsi le canal obligé du 
média, le mécanisme éditorial savamment mis au point à travers les dispositifs de publication et la gestion des droits et autorisations se heurte à des phénomènes d'appropriation individuelle qui se rapprochent d'assez près des pratiques observées dans l'espace de travail «classique » et vis-à-vis desquels l'espace concret, physique, au sein duquel vivent, se déplacent et communiquent les individus conserve toute son importance.

\section{BIBLIOGRAPHIE}

André, J. et Paccaud, A., (2004), Écrire pour l'écran, in : Belisle C. (coord.), La lecture numérique, réalités, enjeux et perspectives, ENSSIB, Lyon, 2004.

Carmes, M., (2002), L'intranet : métaphore des relations de pouvoir dans l'organisation, Actes, $13^{\mathrm{e}}$ colloque de la SFIC, Marseille, Octobre 2002.

Cotte, D., (2005a), Les portails d'entreprise, entre métaphorisation et sémiotisation des organisations, Colloque Culture des organisations et Distic, Nice, 5-6 Décembre 2005.

Cotte, D., (2005b), La structure comme index, Le rôle indiciel des interfaces documentaires, Colloque indice, index, indexation, Lille, 3-4 Novembre 2005.

Despres-Lonnet, M., (2004), Écrits d'écran : le fatras sémiotique, Communication et Langages, $\mathrm{n}$ $\circ 143$.

Despres-Lonnet, M. et Cotte, D., (2005), Usages professionnels des TIC, la textualisation des pratiques, Actes du colloque Enjeux et usages des TIC, Bordeaux, Septembre 2005.

Fischler, C. et Lalhou, S., (1995), « Dossiers, piles d'attente et corbeilles - La gestion quotidienne de l'information au bureau », 58 pages, EDF.

Jeanneret, Y., (2001), Les politiques de l'invisible : du mythe de l'intégration à la fabrique de l'évidence, Document numérique, Hermès sciences publications, vol 5., nº 1-2, 2001.

Jeanneret, Y., (1994), Écrire la science forme et enjeux de la vulgarisation, P.U.F.

Le Marec, J. et Babou, I., (2003), De l'étude des usages à une théorie des « composites » : objets, relations et normes en bibliothèque, in : Souchier et al., Lire, écrire, récrire: Objets, signes et pratiques des médias informatisés, Bibliothèque publique d'information, 2003.

Pedauque, R. et Salaün, J.-M., (2006), Le document à la lumière du numérique, C\&F éditions.

Perriault, J., (1989), La logique de l'usage, Flammarion.

Salaün, J.-M., (2004), Chronique inachevée d'une réflexion collective sur le document, Communication et langages, $\mathrm{n}^{\circ} 140$, Juin 2004.

Souchier, E. et al., (2003), Lire, écrire, récrire : Objets, signes et pratiques des médias informatisés, Bibliothèque publique d'information.

Yates, J., (1982), From Press Book and Pigeonhole to vertical filling : revolution in storage and access systems for correspondance, The journal of business communication, vol. 19. 
Zacklad, M., (2007), Processus de documentarisation dans les Documents pour l'Action (DopA), Babel - edit -, Le numérique : impact sur le cycle de vie du document, ENSSIB - février 2007.

\section{NOTES}

1. Une histoire de l'architecture des espaces de travail, de l'usine du XIX ${ }^{\mathrm{e}}$ siècle au bureau contemporain dépasserait largement le cadre de ce travail, mais il y a là un point de départ qui s'impose d'autant plus que les métaphores utilisées dans les dispositifs informatisés au travail empruntent toutes au vocabulaire (et à l'iconographie) de l'organisation spatiale : portail pour pénétrer dans une application, maison ou home pour la page d'accueil d'un site, bureau pour l'espace de travail sur l'écran, pièces ou espaces de travail dans lesquels on ne pénètre qu'après s'être identifié de la même façon qu'on «badge " pour accéder à tel couloir, emprunter tel ascenseur dans la vie réelle.

2. «Nous nommons architextes (de arkhè, origine et commandement), les outils qui permettent l'existence de l'écrit à l'écran et qui, non contents de représenter la structure du texte, en commandent l'exacution et la réalisation. Autrement dit, le texte naît de l'architexte qui en balise l'écriture » (Souchier et al., 2003).

3. Les thuriféraires de cette pensée sont des auteurs comme Joël de Rosnay, Pierre Lévy, Philippe Quéau, Nicholas Negroponte...

4. Sur tous ces points, il convient de se référer à une recherche collective et interdisciplinaire, réalisée entre 2003 et 2005 au sein du Cnrs sous le pseudonyme collectif de Roger T. Pédauque, concernant le document comme « forme, signe et média », voir bibliographie.

5. Le journal du net donne la définition suivante : «Le portail d'entreprise est, lui, un Intranet qui donne au personnel d'une entreprise et éventuellement à ses partenaires accès, d'une part, à l'ensemble des données et des informations qui appartiennent à l'entreprise en question, que ces données soient structurées (ex.: bases de données internes) ou non (ex.: documents de traitement de texte), et, d'autre part, à une série de sites Web ou de portails verticaux qui se rapportent à la sphère d'activité de l'entreprise ".

6. Le concept de documentarisation, est avancé par Manuel Zacklad, cf. bibliographie.

\section{RÉSUMÉS}

Cet article analyse le rapport du document à l'espace sous deux angles différents et complémentaires. En premier lieu, le document, quelle que soit sa nature, est un espace organisé pour donner du sens à l'information qu'il contient, en second lieu, il est lui-même inséré dans des espaces de classement : boites, rayonnages, pièces, bibliothèques... L'informatique moderne, avec sa logique d'outils intégrés et de portails, aussi bien dans la sphère grand public que dans les entreprises, rassemble sur une même surface (l'écran), et de manière métaphorisée ces deux dimensions.

This paper analyzes the way in which documents and space (especially workspace) are related. Firstly, any document is a typical space, organized in order to produce signification. Secondly, documents are inserted into organizational spaces (boxes, tiers, libraries, etc.), giving them another form of (global) signification. Recent computational applications such as ERP (Enterprise 
Resource Planning) and portals assemble these different elements on the screen as a metaphor for real space.

\section{INDEX}

Mots-clés : document, portail d'entreprise, espace de travail, sémiotique

Keywords : document, enterprise portal, workspace, semiotics

\section{AUTEUR}

\section{DOMINIQUE COTTE}

Dominique Cotte est maître de conférences en sciences de l'information et de la communication, à partir d'un parcours disciplinaire en philosophie (Dea, 1977) et en Sic (Dess, 1978 ; Doctorat, 1999) et un parcours professionnel réalisé autour des lieux et des technologies du traitement de l'information (presse, publicité, conseil). Ses travaux de recherche porte sur l'émergence et la transformation des formes médiatiques dans divers contextes qui vont des entreprises centrées sur le traitement de l'information, comme les rédactions des journaux, aux entreprises généralistes et aux outils qui s'y déploient (intranets, portails d'entreprise...). Il enseigne en master « Sciences de l'information et du document » à l'Université de Lille 3, ainsi que dans d'autres formations en Sic. Adresse électronique : dominique.cotte@univ-lille3.fr. 